\title{
46. PHOTOMETRIC INVESTIGATION OF THE ASSOCIATION CAR OB 2
}

\author{
W. SEGGEWISS \\ Observatorium Hoher List der Universitäts-Sternwarte Bonn, Germany
}

\begin{abstract}
A photometric investigation of the association Car OB 2 was carried out with a photoelectric standard sequence observed with the ESO photometric telescope and plates taken with the $\mathrm{ADH}$ telescope of Boyden Observatory. The distance of Car OB 2 is $2.04 \mathrm{kpc}$, the color excess $E_{\mathrm{B}-\mathrm{v}}$ is $0^{\mathrm{m}} .38$. The main sequence of this 'OB' association reaches at least to late A-type stars. The luminosity function was determined and compared with the luminosity functions of very young open clusters observed by Walker (1957). The distance of a group of 13 early-type stars in the area of Car OB 2 ('group c') was found to be $2.5 \mathrm{kpc}$, the reddening $0^{\mathrm{m}} .68$. The relation of Car OB 2 and group $\mathrm{c}$ to the structure of the Carina arm is regarded.
\end{abstract}

The association Car OB 2 is marked by a group of brilliant blue stars which are distributed over a nearly elliptical ring. The major axis of the ellipse is approximately $30 \mathrm{~min}$, the minor axis $23 \mathrm{~min}$ of arc. The center lies a half degree above the galactic plane near the galactic longitude of $290^{\circ}$. The precise coordinates are

$$
\begin{array}{lll}
\alpha=11^{\mathrm{h}} 03^{\mathrm{m}} .7 & & l^{\mathrm{II}}=290.0 \\
\delta=-59^{\circ} 31^{\prime} & (1950) & b^{\mathrm{II}}=+0.4 .
\end{array}
$$

This remarkable compact group of early-type stars has been the subject of some photometric and spectroscopic studies: In 1952 Bok and van Wijk (1952) observed four stars of the group in the PV-system (HD 96446, 96622, 96638, 96670). They also were the first who qualified the group as an association. Unfortunately they had only Henry Draper spectral types at their disposal with which to derive a distance of $1.4 \mathrm{kpc}$. Therefore Hoffleit (1956) determined MKK spectral types for five stars in Car OB 2 (HD 96248 B 1 I, 96446 B 2 V, 96622 O 9.5 IV, 96638 O 8, 96670 O 8). Together with the photoelectric magnitudes and colors in the Bok-van Wijk system she derived a distance of $1.7 \mathrm{kpc}$. But if one excludes HD 96446 ('exceptionally nearby for group membership', Hoffleit) one derives $2.0 \mathrm{kpc}$ as the mean distance.

Feinstein (1964) observed 27 stars in the UBV-system. He used the main-sequence fitting-method and found a distance of $1.2 \mathrm{kpc}$ and a color excess $E_{\mathrm{B}-\mathrm{v}}=0.40$. He also noticed a second group ('group b') of stars in the field of the association with a distance of $0.4 \mathrm{kpc}$ and a reddening of 0.15 .

Early in 1969 the author observed a photoelectric standard sequence of 25 stars in the UBV-system with the $1 \mathrm{~m}$-telescope of the European Southern Observatory in Chile. The limiting magnitude was 14.2 in V. The plates had been taken by H. Haffner during his stay at Boyden Observatory in 1958. 480 stars were measured on four plates in each color with a Becker-type iris photometer of Hoher List Observatory. The more interesting blue parts of the two-color diagram and the color-magnitude diagrams are shown in Figures 1 and 2. The main-sequence of the association is clearly recognizable. The lefthand dashed line in Figure 1 and the solid lines in Figure 


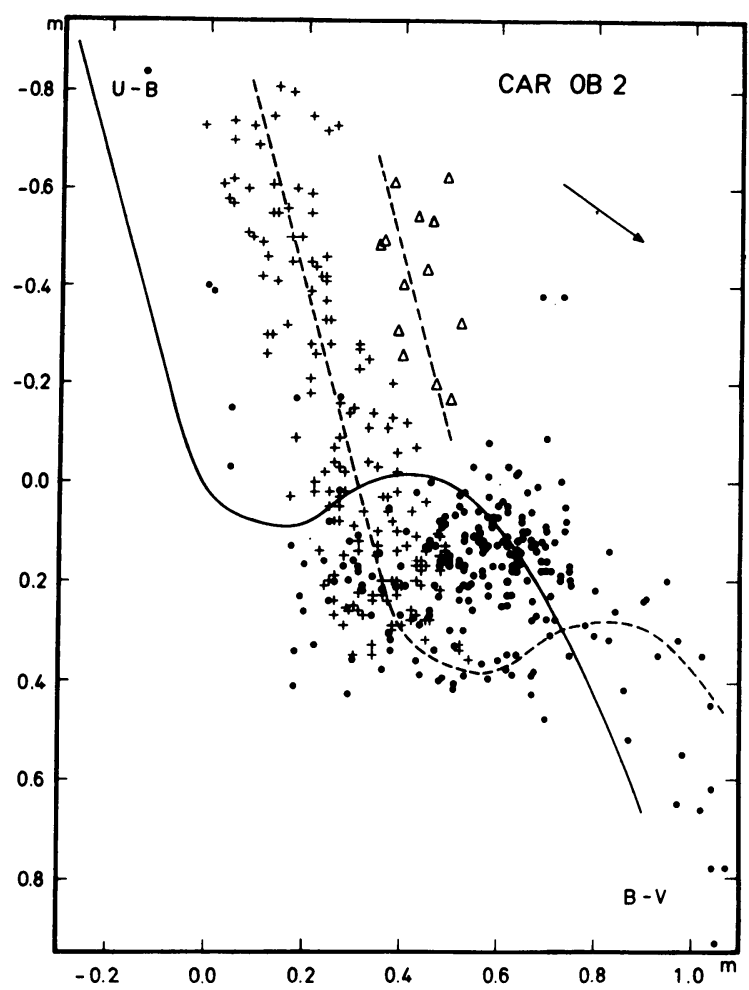

Fig. 1. Two-color diagram of the association Car OB 2. + probable members, field stars, $\triangle$ stars of group c.
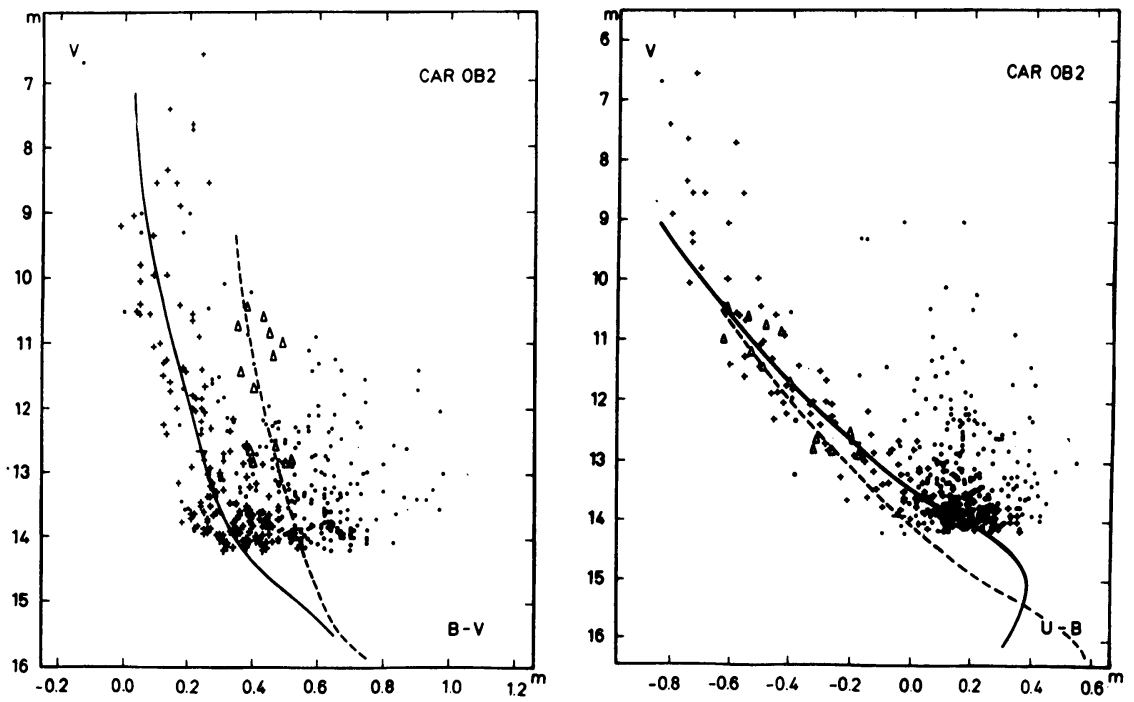

Fig. 2. Color-magnitude diagrams of the association Car OB 2. + probable members, stars, $\triangle$ stars of group $\mathrm{c}$. 
2 are the fitted ZAMS. In order to separate the association members from nonmembers, all stars nearer to the superimposed ZAMS than two times the mean internal error of the photometry $(\sigma=+0.04$ in $V, 0$.05 in B-V, and 0.07 in U-B) were selected. These probable members are indicated by crosses. The color excess $E_{\mathrm{B}-\mathrm{v}}$ equals 0.38 , the distance modulus not corrected for absorption equals 12.65 . Under the assumption $R=3.0$ the distance was determined to be $2.04 \mathrm{kpc}$, the same value that Hoffleit derived. The linear dimensions of Car OB 2 are therefore 18 by $13 \mathrm{pc}$. The discrepancy between our distance and that of Feinstein is easily explained: If one has only those stars brighter than the 11 th mag. and only the long wavelength color-magnitude diagram at one's disposal it is impossible to fit the main-sequence in a correct way. UBV data for fainter stars as well and the short wavelength colormagnitude diagram with its more steeply inclined upper part of the main-sequence are needed.

It is worthwhile to point out that Car OB 2 not only consists of $\mathrm{O}$ and early $\mathrm{B}$ stars - as the name 'OB' association may suggest - but that there is a continuous transition to later B- and A-type stars. The same was found by Seggewiss (1968) in the northern part of the association Sco OB 1 (= Tr 24 and its subgroups).

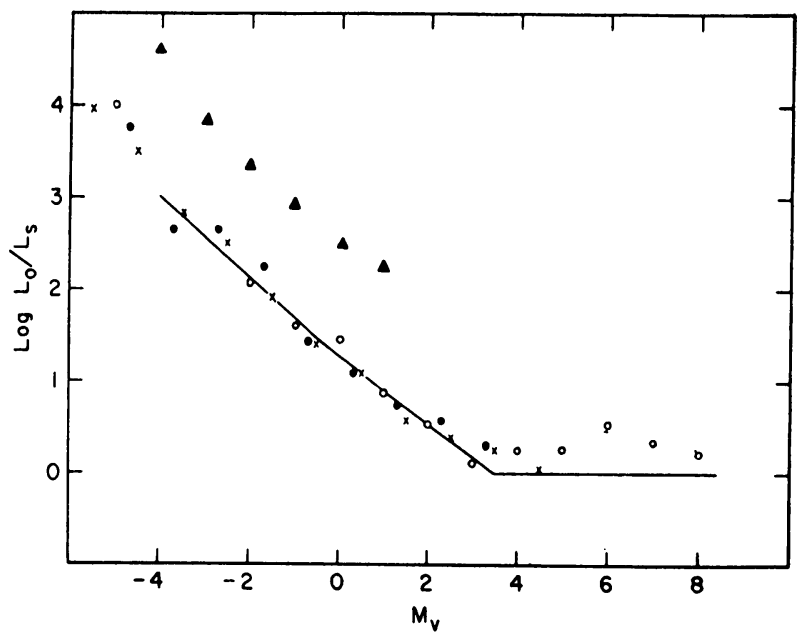

Fig. 3. Luminosity function of Car $O B$ compared with the luminosity functions of young open clusters as derived by Walker (1957). $\triangle$ Car OB 2, NGC 6530, ONGC 2264, $\times$ Orion nebula cluster.

The luminosity function of Car OB 2 has the same slope as the initial luminosity function derived by Salpeter (1955) and the luminosity functions of very young open clusters observed by Walker (1957). Figure 3 was taken from Walker's paper. The ordinate is the logarithm of the ratio of the observed luminosity functions $L_{o}$ to the luminosity function $L_{s}$ of main-sequence stars in the solar neighborhood. The line indicates Salpeter's initial luminosity function. Dots refer to NGC 6530, open circles to NGC 2264, crosses to the Orion nebula cluster (star numbers adjusted). The 
luminosity function of $\mathrm{Car} \mathrm{OB} 2$ is represented by triangles (star numbers not adjusted).

A group of 13 highly reddened stars, marked by triangles, can be distinguished in Figure 1. It is surprising to notice that all 13 stars lie within a small area, only $8 \mathrm{~min}$ of arc diameter, in the field of the association. One does not find any indication that the interstellar absorption in the field of Car OB 2 is non-uniform. Therefore one may conclude that these early-type stars form a separate group c, more reddened than Car OB $2\left(E_{\mathrm{B}-\mathrm{v}}=0.68\right)$ and more distant ( $2.5 \mathrm{kpc}$, fitted ZAMS as dashed lines in Figure 2).

Probable field stars in the area of $\mathrm{Car}$ OB 2, mostly weak reddened A- and F-type stars, are indicated by dots (Figures 1 and 2). Only very few stars form the upper part of Feinstein's group b (Figure 1) which therefore consists probably only of field stars. The bluest star is the helium star HD 96446 investigated by Jaschek and Jaschek (1959).

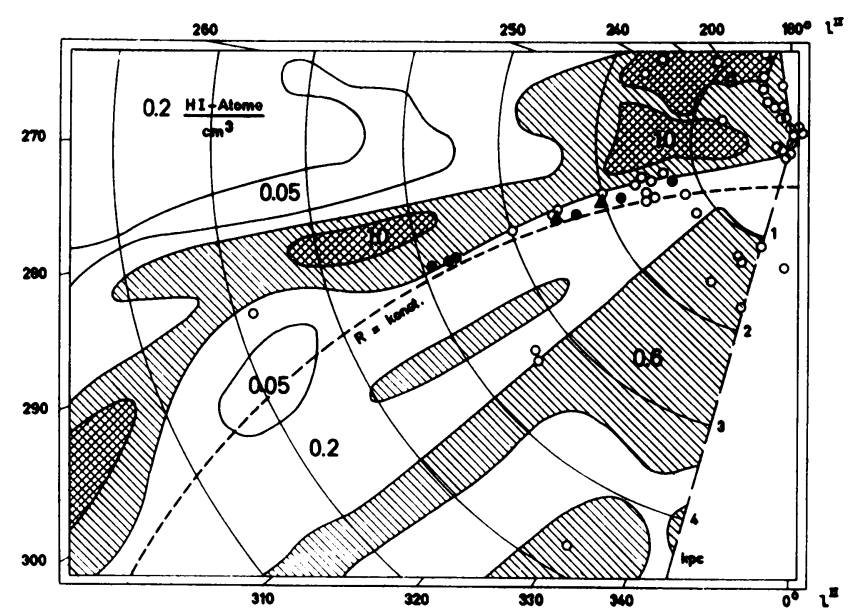

Fig. 4. Galactic plane around $l I I=290^{\circ}$. $\triangle$ Car OB 2 and group c, young open clusters and stellar groups investigated by Schmidt and Diaz Santanilla (1964), $\bigcirc$ open clusters and H II regions.

Figure 4 shows the galactic plane after a drawing by Beer (1961). The density of neutral hydrogen atoms is indicated by different types of hatching. Small circles denote open clusters and $\mathrm{H}$ II regions. Car OB 2 and group c are represented by triangles. The dots indicate 6 young open clusters and stellar groups in the vicinity of Car OB 2, which were investigated by Schmidt and Diaz Santanilla (1964) of Hoher List Observatory with the plates now used for Car OB 2. The earliest spectral types, distances and reddening of these clusters together with the corresponding values of Car OB 2 and group c are given in the Table $\mathrm{I}$.

Two points are to be stressed: First, the chain of spiral arm tracers does not coincide with the maximum density of neutral hydrogen. Second, the interstellar reddening is surprisingly low, only 0.5 to a distance of $4 \mathrm{kpc}$, though we are looking lengthwise through a spiral arm.

Finding charts and UBV data of all 480 stars are available upon request from Hoher List Observatory. 
TABLE I

Earliest spectral types, distances and color excesses of young open clusters and stellar groups in the vicinity of Car OB 2

\begin{tabular}{|c|c|c|c|}
\hline $\begin{array}{l}\text { Object } \\
\text { near } l=290^{\circ}\end{array}$ & Sp. Type & $\begin{array}{l}d \\
\mathrm{kpc}\end{array}$ & $\begin{array}{l}E_{\mathrm{B}-\mathrm{v}} \\
\mathrm{m}\end{array}$ \\
\hline NGC 3572 a 1 & B I IV-V & 1.24 & 0.26 \\
\hline $3572 \mathrm{a} 2$ & B 1 IV-V & 3.65 & 0.51 \\
\hline $3572 \mathrm{~b}$ & $09 \mathrm{~V}$ & 3.90 & 0.52 \\
\hline $3590 a$ & B $5 \mathrm{~V}$ & 1.82 & 0.19 \\
\hline $3590 \mathrm{~b}$ & B2 IV & 3.70 & 0.44 \\
\hline $\operatorname{Tr} \quad 18$ & B4 V & 2.33 & 0.37 \\
\hline Car OB 2 & 08 & 2.04 & 0.38 \\
\hline 'Group c’' & 07 & 2.50 & 0.68 \\
\hline
\end{tabular}

\section{References}

Beer, A.: 1961, Monthly Notices Roy. Astron. Soc. 123, 191.

Bok, B. J. and van Wijk, U.: 1952, Astron. J. 57, 213.

Feinstein, A.: 1964, Observatory 84, 111.

Hoffleit, D.: 1956, Astrophys. J. 124, 61.

Jaschek, M. and C.: 1959, Publ. Astron. Soc. Pacific 71, 465.

Salpeter, E. E.: 1955, Astrophys. J. 121, 161.

Schmidt, H. and Diaz Santanilla, G.: 1964, Veröffent. Astron. Inst. Univ. Bonn, 71.

Seggewiss, W.: 1968, Z. Astrophys. 68, 142.

Walker, M. F.: 1957, Astrophys. J. 125, 636. 